\title{
Mda-7/IL-24 enhances sensitivity of $B$ cell lymphoma to chemotherapy drugs
}

\author{
MING MA ${ }^{1}$, LIANMEI ZHAO ${ }^{2}$, GUOGUI SUN ${ }^{3}$, CHAO ZHANG $^{2}$, LIHUA LIU $^{2}$, \\ YANYAN DU ${ }^{1}$, XINGXIAO YANG ${ }^{4}$ and BAOEN SHAN ${ }^{2}$
}

\author{
${ }^{1}$ Clinical Laboratory and ${ }^{2}$ Research Center, The Fourth Hospital of Hebei Medical University, Shijiazhuang, Hebei 050011; \\ ${ }^{3}$ Department of Tumor Research Institute, Tangshan People's Hospital, Tangshan, Hebei 063000; \\ ${ }^{4}$ Department of Infection Management, The Fourth Hospital of Hebei Medical University, \\ Shijiazhuang, Hebei 050011, P.R. China
}

Received November 6, 2015; Accepted December 15, 2015

DOI: $10.3892 / o r .2016 .4622$

\begin{abstract}
Interleukin-24 (IL-24) is a cytokine encoded by a tumor suppressor gene of the IL-10 family, also known as the melanoma differentiation associated gene-7 (Mda-7) and first discovered in human melanoma cells. Mda-7/IL-24 has been shown to inhibit the proliferation of various human tumor cell lines, but its effect on the sensitivity of B cell lymphoma to chemotherapy agents is not yet clear. The present study investigated the effects of Mda-7/IL-24 overexpression on the sensitivity of human B cell lymphoma cells to chemotherapy, as well as its mechanism of action. The sensitivity of stable Mda-7/IL-24 overexpressing Raji and Daudi cells to cis-diamminedichloroplatinum (CDDP), epirubicin and vinblastine (VCR) were assessed by the MTS method, and the $\mathrm{IC}_{50}$ value calculated. Cell apoptosis and the intracellular accumulation of Rhodamine-123 were assayed by flow cytometry. The expression of multidrug resistance gene 1 (MDR1), B-cell-specific Moloney murine leukemia virus insertion site 1 (BMI1), topoisomerase II (Topo II) and multidrug resistance-related protein 1 (MRP1) mRNA and protein were analyzed by reverse transcription quantitative polymerase chain reaction (RT-qPCR) and western blotting, respectively. In addition, western blot analysis was also used to investigate the effect of Mda-7/IL-24 on activity of GTP-RhoA-ERK signaling pathway in Raji and Daudi cells. Growth inhibition
\end{abstract}

Correspondence to: Professor Baoen Shan, Research Center, The Fourth Hospital of Hebei Medical University, 12 Jiankang Road, Shijiazhuang, Hebei 050011, P.R. China

E-mail: shanbaoen@163.com

Abbreviations: Mda-7/IL-24, melanoma differentiation associated gene-7/interleukin-24; CDDP, cis-diamminedichloroplatinum; VCR, vinblastine; $\mathrm{IC}_{50}, 50 \%$ inhibiting concentration; MDR, multidrug resistance

Key words: Mda-7/IL-24, chemotherapy sensitivity, B-cell lymphoma, multidrug resistance and apoptosis rates of Mda-7/IL-24 overexpressing Raji and Daudi cells were higher than those of non-transfected cells and cells transfected with vector alone when treated with CDDP, epirubicin and VCR. The $\mathrm{IC}_{50}$ values of CDDP, epirubicin and VCR were lower for Mda-7/IL-24-overexpressing Raji and Daudi cells than for non-transfected cells and cells transfected with empty vector. Intracellular accumulation of Rhodamine-123 and the expression of Topo II were higher, while the levels of MDR1, BMI and MRP1 mRNA and protein were lower, in Mda-7/IL-24 overexpressing Raji and Daudi cells. Furthermore, the activities of GTP-RhoA-ERK signaling pathway in Raji and Daudi cells were suppressed. These results indicated that Mda-7/IL-24 enhanced the sensitivity of B lymphoma cells to chemotherapy agents by altering the expression of multidrug-resistance genes via downregulating GTP-RhoA-ERK signaling pathway, suggesting that treatment of B cell lymphomas with Mda-7/IL-24 could avoid MDR.

\section{Introduction}

Lymphoma is a common hematopoietic malignancy originating from lymphoid system. Based on their immunophenotypes, most lymphomas are B cell type (1). Although chemotherapy remains the treatment of choice for $B$ cell lymphoma, the main barrier to its success is the development of multidrug resistance (MDR), by which tumors become insensitive to multiple chemotherapeutic agents after exposure to one (2). Tumor development of MDR is thought to be due to their overexpression of MDR-related genes. Several novel factors, including a defective apoptosis pathway, the pump effect of MDR-related proteins on drugs, and enhanced DNA repair activity, have been found to play critical roles in the development of MDR. Since MDR is a primary cause of the clinical failure of chemotherapy, there is an urgent need to identify novel and more effective strategies to prevent drug resistance. Investigation of the mechanisms underlying MDR may identify chemosensitizers for clinical application in patients with B cell lymphoma.

Interleukin-24 (IL-24), a novel member of the IL-10 family of cytokines also called melanoma differentiation associated gene-7 (Mda-7), was first identified in human melanoma 
cells (3-5). IL-24 expression was found to be lost in a broad spectrum of malignant tumors, including hematopoietic malignancies, whereas overexpression of this gene resulted in ubiquitous growth inhibition, induction of apoptosis, reversal of malignant phenotypes and terminal differentiation in a variety of tumors (5-7). Recently, overexpression of Mda-7/IL-24 was reported to result in the apoptosis of various cancer cells, including lung, hepatoma, pancreatic, breast, melanoma and prostatic carcinoma, but to have no harmful effects on normal cells (7-9). Mda-7/IL-24 was found to reverse MDR and enhance sensitivity to chemotherapy drugs of human colorectal and hepatocellular carcinomas. This ability of Mda-7/IL-24 to reverse MDR in solid tumors led to our hypothesis, that overexpression of Mda-7/IL24 may sensitize human B lymphoma cells to chemotherapy.

The present study reports, for the first time, that Mda-7/ IL-24 sensitized B lymphoma cells lines to treatment with the chemotherapy agents cis-diamminedichloroplatinum (CDDP), epirubicin and vinblastine (VCR) resulting in significantly higher percentages of apoptotic cells. The $50 \%$ inhibitory concentrations $\left(\mathrm{IC}_{50}\right)$ of these chemotherapy drugs were significantly lower in lymphoma cells overexpressing Mda-7/IL-24 than in those that were not. Moreover, the expression of multidrug resistance protein 1 (MDR1), multidrug resistance-related protein 1 (MRP1), and B-cell-specific moloney murine leukemia virus insertion site 1 (BMI1) was lower, whereas topoisomerase II (Topo II) expression was higher, in lymphoma cells overexpressing Mda-7/IL-24. Furthermore, the activities of ERK signaling pathway in Raji and Daudi cells overpressing Mda-7/IL-24 were suppressed. These results suggest that the combination of chemotherapy drugs and Mda-7/IL-24 may be a potential clinical strategy to avoid MDR in patients with $\mathrm{B}$ cell lymphoma.

\section{Materials and methods}

Cell lines and regents. The human B lymphoma cells Raji and Daudi were obtained from the Research Center of the Fourth Hospital of Hebei Medical University (Hebei, China) and cultured in RPMI-1640 medium (Sigma, St. Louis, MA, USA) supplemented with $10 \%$ fetal calf serum (FCS; Gibco, Grand Island, NY, USA) in a $5 \% \mathrm{CO}_{2}$ humidified incubator at $37^{\circ} \mathrm{C}$. MTS and Rhodamine- 123 were obtained from Sigma. Annexin V-FITC and 7-AAD double stain kit was purchased from BD Pharmingen (San Diego, CA, USA). Antibodies to total p44/42 MAPK (ERK 1/2), pho-p44/42 MAPK (p-ERK 1/2) and GTP-RhoA were all purchased from Cell Signaling Technology, Inc. (Beverly, MA, USA). Antibodies against Mda-7/IL-24, P-gp, MRP1, BMI1, Topo II and $\beta$-actin were purchased from Abcam (Cambridge, MA, USA). TRIzol reagent was purchased from Invitrogen (Carlsbad, CA, USA). GoTaq $^{\circledR}$ qPCR Master Mix was purchased from Promega (Madison, WI, USA). RevertAid ${ }^{\mathrm{TM}}$ First Strand cDNA Synthesis kit was purchased from MBI Fermentas (Hanover, MD, USA). CDDP was purchased from Qilu Pharmaceutical Co., Ltd. Shandong, China (lot no. 1WA2A1501008). Epirubicin was purchased from Hisun Pharmaceutical Inc., Zhejiang, China (lot no. 1140102A). VCR was purchased from Shenzhen Main Luck Pharmaceuticals Inc., Guangdong, China (lot no. 1406V3).
Transfection of lymphoma cells. Raji and Daudi cells were transfected with the Mda-7/IL-24 gene linked to a lentiviral vector. Briefly, Raji and Daudi cells were cultured with supernatants of 293T cells producing lentiviral vector encoding the human Mda-7/IL-24 gene and selected in the presence of G418 $(500 \mu \mathrm{g} / \mathrm{ml})$. As a control, Raji and Daudi cells were transfected with vector alone.

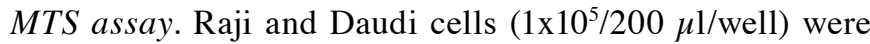
seeded into 96-well plates (Gibco). After $24 \mathrm{~h}, 10 \mu \mathrm{l}$ MTS solution $[5 \mathrm{mg} / \mathrm{ml}$ in phosphate-buffered saline (PBS)] were added to each well and the plates incubated for $4 \mathrm{~h}$. The absorbance value (OD value) of each well was measured at $492 \mathrm{~nm}$ on an ELISA microplate reader set. The inhibition rate (\%) of chemotherapy drugs was calculated as: [(OD value control group - OD value experimental group)/OD value control group] x $100 \%$.

Flow cytometry. To investigate apoptosis, single-cell suspensions of Raji and Daudi cells $\left(1 \times 10^{6}\right.$ cells/sample) were resuspended in ice-cold PBS, with the volume adjusted to $1 \mathrm{ml}$ with PBS. The cells were incubated with Annexin V-FITC and 7-AAD double stain according to the manufacturer's instructions and analyzed using a flow cytometer (FACSCalibur ${ }^{\mathrm{TM}}$; Becton-Dickinson, USA). Data were analyzed with CellQuest Pro software (FACSCalibur ${ }^{\mathrm{TM}}$; BD) and expressed as the mean \pm standard error of the mean of three independent experiments.

Western blot analysis. Raji and Daudi cells were lysed with $250 \mu \mathrm{l}$ of lysis buffer (1\% Triton X-100, $150 \mathrm{mM} \mathrm{NaCl}$, $10 \mathrm{mM}$ Tris-HCl, pH 7.4, $1 \mathrm{mM}$ EDTA, $1 \mathrm{mM}$ EGTA, $\mathrm{pH} 8.0,0.2 \mathrm{mM} \mathrm{Na} \mathrm{VO}_{4}, 0.2 \mathrm{mM}$ phenylmethylsulfonylfluride and $0.5 \% \mathrm{NP}-40$ ). The lysates were subjected to sodium dodecyl sulfate-polyacrylamide gel electrophoresis (SDS-PAGE) and were electrotransferred onto a polyvinylidene difluoride membrane. The membranes were incubated in PBS containing 5\% bovine serum albumin for $2 \mathrm{~h}$ at room temperature, followed by overnight incubation at $4{ }^{\circ} \mathrm{C}$ with 1:1,000 dilutions of the primary antibodies, including antibodies to Mda-7/IL-24, P-gp, MRP1, BMI1, Topo II, GTP-RhoA, Total-ERK1/2, p-ERK1/2 and $\beta$-actin Ab. The membranes were developed with the Odyssey infrared imaging system according to the manufacturer's instructions. The levels of protein in each sample were normalized relative to those of $\beta$-actin. Each experiment contained triplicate wells of each sample and all experiments were repeated at least three times.

$R N A$ preparation and reverse transcription quantitative polymerase chain reaction ( $R T-q P C R)$ analysis. Total RNA was extracted from Raji and Daudi cells using TRIzol reagent according to the manufacturer's directions. RNA concentration was routinely measured spectrophotometrically and its quality was evaluated by visualization after agarose gel electrophoresis and ethidium bromide staining. First strand cDNAs were generated from one microgram aliquots of total RNA using RevertAid ${ }^{\mathrm{TM}}$ First Strand cDNA Synthesis kit and incubation at $42^{\circ} \mathrm{C}$ for $60 \mathrm{~min}$. The resultant cDNAs were amplified by RT-qPCR using GoTaq ${ }^{\circledR}$ qPCR Master Mix and 

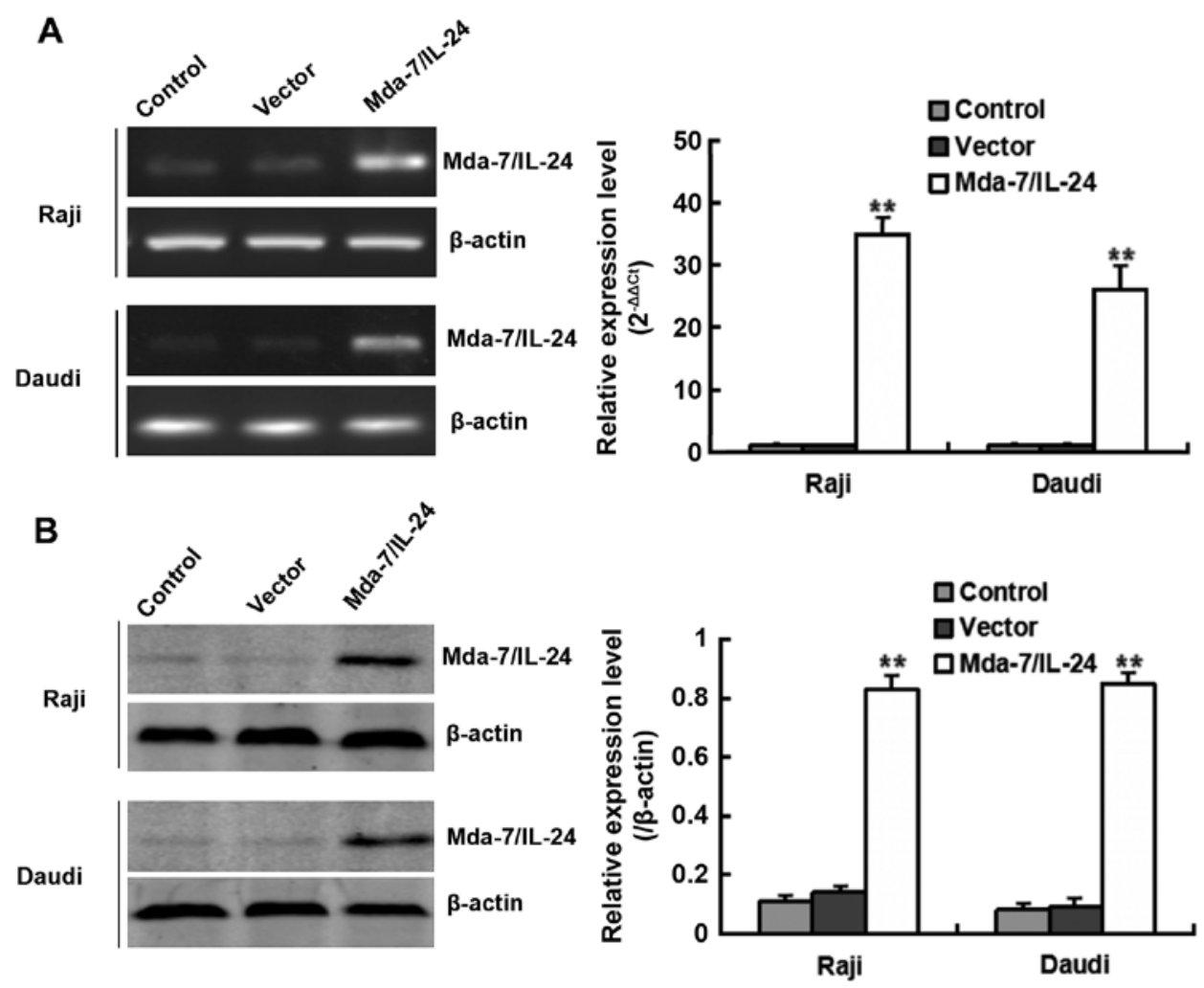

Figure 1. Levels of expression of Mda-7/IL-24 in different clones of stably transfected Raji and Daudi cells. Control groups, parent cell lines; vector groups, cells stably transfected with vector alone; Mda-7/IL-24 groups, cells stably transfected with vector expressing Mda-7/IL-24. (A) RT-qPCR analysis of Mda-7/ IL-24 mRNA expression. (B) Mda-7/IL-24 protein expression analyzed by western blotting; blots were subsequently incubated with antibody to $\beta$-actin as a loading control. The results shown are representative of three independent experiments with similar results. ${ }^{* *} \mathrm{P}<0.01$ compared with control and vector groups.

Table I. Primer sequences for the reverse transcription quantitative polymerase chain reaction.

\begin{tabular}{llc}
\hline Gene & \multicolumn{1}{c}{ Primer sequence } & $\left({ }^{\circ} \mathrm{C}\right)^{\mathrm{a}}$ \\
\hline Mda-7/IL-24 & F 5'-CGACAGCCTCTCAAATGCAG-3' & 60 \\
& R 5'-GCTCTCCGGAATAGCAGAAACC-3' & \\
\multirow{2}{*}{$\beta$-actin } & F 5'-GTTGTGATGGGTTCTGA-3' & 60 \\
& R 5'-GAGCAATAGCGTCTGTG-3' & \\
MRP1 & F 5'-CGCTGAGTTCCTGCGTACC-3' & 60 \\
& R 5'-TCTGCGGTGCTGTTGTGG-3' & \\
MDR1 & F 5'-CAGAGGGGATGGTCAGTGTT-3' & 60 \\
& R 5'-CGTGGTGGCAAACAATACAG-3' & \\
BMI1 & F 5'-AAATGCTGGAGAACTGGAAAG-3' & 60 \\
& R 5'-AACTGTGGATGAGGAGACTG-3' & \\
Topo II & F 5'-GGCTCGATTGTTATTTCCAC-3' & 60 \\
& R 5'-GGTTGTAGAATTAAGAATAGC-3' & \\
\hline
\end{tabular}

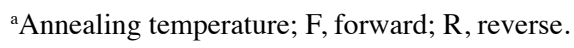

specific primers for MDR1, MRP1, BMI1, Topo II and $\beta$-actin (Shanghai Generay Biotech; Table I), with the latter used as an internal control. PCR products were separated on $2 \%$ agarose gels and visualized by ethidium bromide staining. The relative level of expression of each target gene was assessed by the
$2^{-\Delta \Delta \mathrm{Ct}}$ method, where $\Delta \Delta \mathrm{Ct}=(\mathrm{Ct}$ target gene of experimental group - $\mathrm{Ct} \beta$-actin of experimental group) - ( $\mathrm{Ct}$ target gene of control group - $\mathrm{Ct} \beta$-actin of control group).

Intracellular accumulation of Rhodamine-123 in lymphoma cells. To assess the efflux of MDR-related proteins, Rhodamine-123 exclusion assays were performed. Single-cell suspensions of Raji and Daudi cells were resuspended in ice-cold PBS, washed twice with PBS and co-cultured with Rhodamine-123 (5 mg/ml) for $35 \mathrm{~min}$. The cells were again washed and analyzed using a FACScan flow cytometer to determine the intensity of fluorescence. Data were analyzed by CellQuest Pro software.

Statistical analysis. All statistical analyses were performed using SPSS 13.0 software (SPSS, Inc., Chicago, IL, USA). Differences were assessed by one-way analysis of variance, with Student's t-tests used to compare two independent samples. $\mathrm{P}<0.05$ was considered statistically significant. The $\mathrm{IC}_{50}$ values of Raji and Daudi cells were calculated by origin 5 software (OriginLab, Northampton, MA, USA). All data are expressed as the mean \pm standard deviation. Results shown in the images are representative of at least three independent experiments.

\section{Results}

Expression of Mda-7/IL-24 in human Raji and Daudi lymphoma cell lines. Mda-7/IL-24 mRNA and protein were 
A
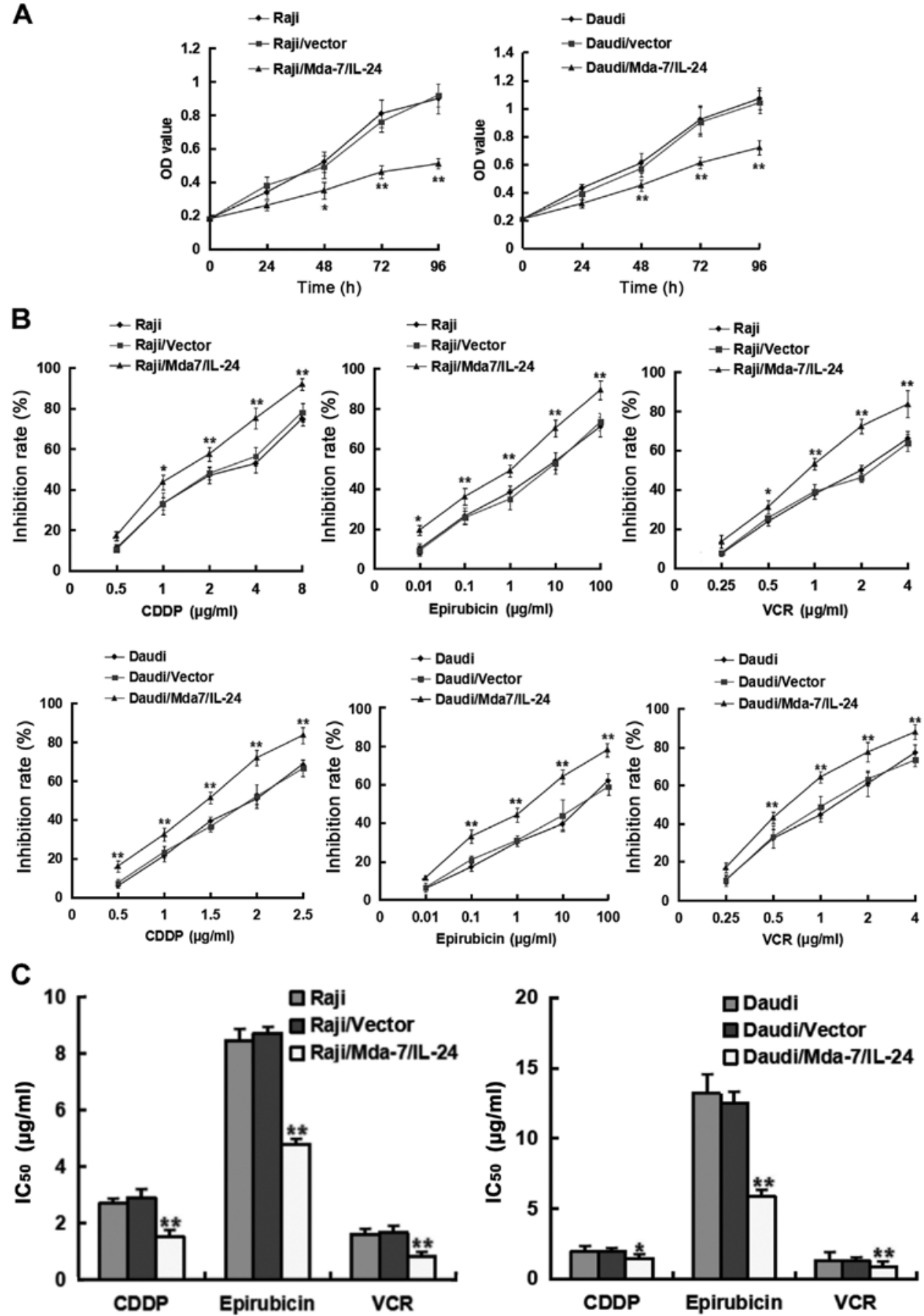

Figure 2. Ability of Mda-7/IL-24 to retarded proliferation and enhance the chemosensitivity of Raji and Daudi cells to anticancer drugs. (A) The significantly lower absorbance value (OD value) was observed in Raji and Daudi cells overexpressing Mda-7/IL-24 than non-transfected cells and cells transfected with vector alone for 48,72 and $96 \mathrm{~h}$, and there were no significant differences between the latter two cultures. (B) The chemotherapy drugs CDDP, epirubicin and VCR dose-dependently inhibited lymphoma cell proliferation over $24 \mathrm{~h}$. Cells overexpressing Mda-7/IL-24 were more sensitive to chemotherapy drugs than non-transfected and vector-transfected cells. (C) IC $_{50}$ values of CDDP, epirubicin and VCR were lower in Raji and Daudi cells transfected with Mda-7/IL-24 than in non-transfected and vector-transfected cells $(\mathrm{P}<0.05)$. Absorbance values and $\mathrm{IC}_{50}$ values are presented as the means $\pm \mathrm{SD}$ from three independent experiments. ${ }^{*} \mathrm{P}<0.05,{ }^{* *} \mathrm{P}<0.01$ compared with control and vector groups.

assayed in parent Raji and Daudi cells (control), in cells transfected with vector alone, and in cells overexpressing Mda-7/IL-24 by RT-qPCR and western blotting, respectively. The expression of Mda-7/IL-24 mRNA and protein was weakly detectable in control cells and cells transfected with empty vector. In contrast, Mda-7/IL-24 overexpressing cell lines showed significantly increased expression of both Mda-7/IL-24 mRNA and protein (Fig. 1).
Mda-7/IL-24 retards proliferation and enhances the sensitivity of B lymphoma cells to chemotherapeutic agents. To investigate the effect of Mda-7/IL-24 on proliferation and chemotherapy sensitivity of B lymphoma cells, MTS assays were performed. As shown in Fig. 2A, transfection with Mda-7/IL-24 significantly induced growth suppression in Raji and Daudi cells overexpressing Mda-7/IL-24, compared with non-transfected cells and cells transfected with vector alone. 

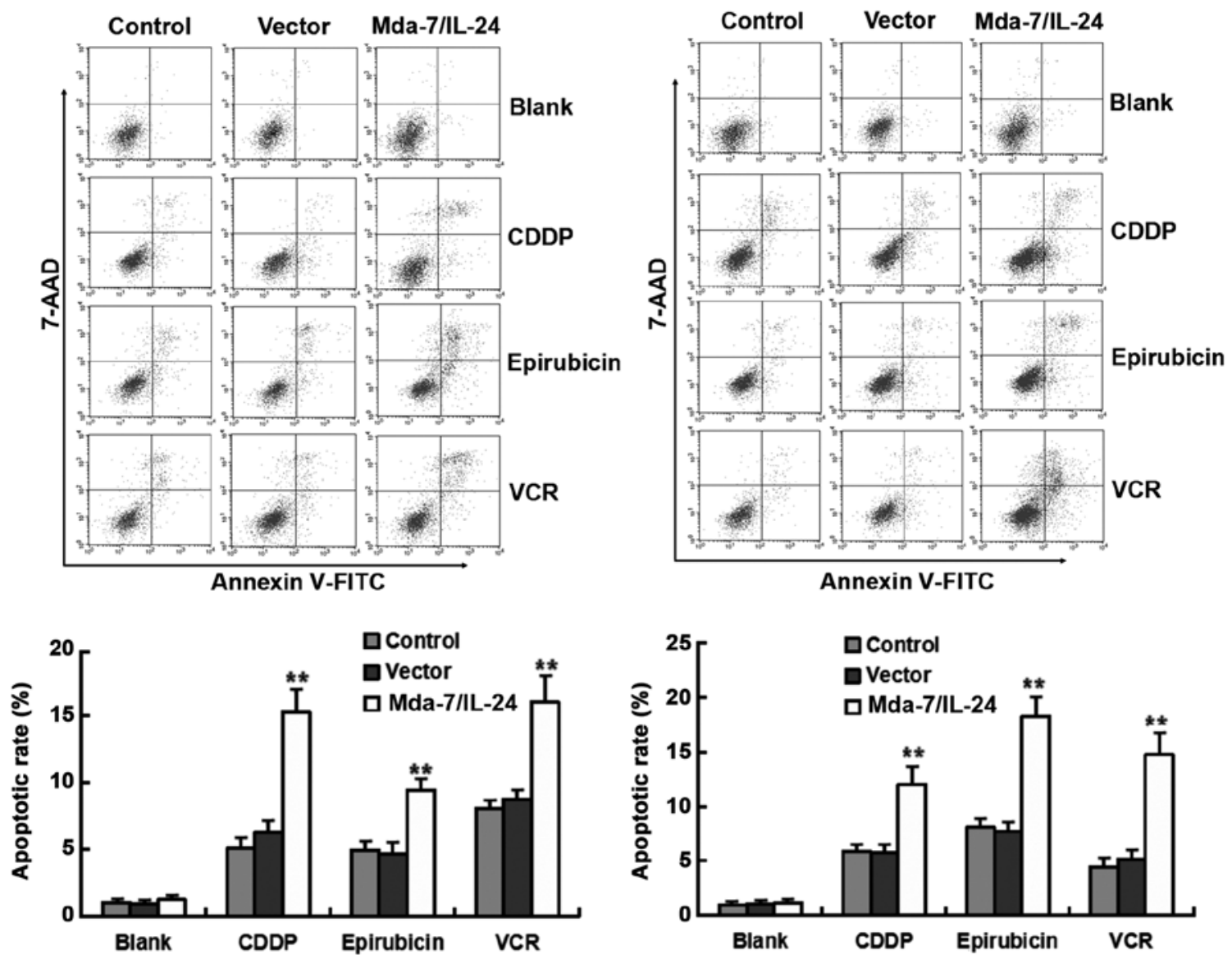

Figure 3. Induction of apoptosis by different chemotherapy drugs in stably transfected lymphoma cells. Raji and Daudi cells were treated without chemotherapy agent or with CDDP $(0.5 \mu \mathrm{g} / \mathrm{ml})$, epirubicin $(0.25 \mu \mathrm{g} / \mathrm{ml})$ or VCR $(0.01 \mu \mathrm{g} / \mathrm{ml})$ for $24 \mathrm{~h}$. There was no difference in the percentage of Annexin V-FITC-positive cells between the cell lines of Raji and Daudi with and without Mda-7/IL-24 expression, when the cells were not treated with chemotherapy agent. However, when Raji and Daudi cells were treated with CDDP, epirubicin or VCR, the percentage of Annexin V-FITC-positive cells were significantly higher in cells transfected with Mda-7/IL-24 than in non-transfected and vector-transfected cells $(\mathrm{P}<0.01)$, and there was no significant difference between the latter two groups $(\mathrm{P}>0.05)$. Similar results were obtained in three independent experiments. ${ }^{* *} \mathrm{P}<0.01$, compared with the control and vector groups.

We further explored whether Mda-7/IL-24 had chemosensitizing effects on B lymphoma cells. As shown in Fig. 2, the proliferation of the two lymphoma cell lines over $24 \mathrm{~h}$ was dose-dependently inhibited by CDDP, epirubicin and VCR, with significantly higher inhibition observed in Raji and Daudi cells overexpressing Mda-7/IL-24 than in non-transfected cells and cells transfected with vector alone (Fig. 2A and B). The $\mathrm{IC}_{50}$ values of CDDP, epirubicin and VCR were lower in cells overexpressing Mda-7/IL-24 than in non-transfected cells and cells transfected with empty vector (Fig. 2C). These results suggested that Mda-7/IL-24 can retard proliferation of B lymphoma cells, and enhance the sensitivity of B lymphoma cells to chemotherapy drugs.

To further determine the potential chemosensitizing effects of Mda-7/IL-24, flow cytometry was performed to investigate whether overexpressing Mda-7/IL-24 induced Raji and Daudi cell apoptosis. As shown in Fig. 3, no difference in the percentage of Annexin V-FITC-positive cells was observed between the cell lines of Raji and Daudi with and without Mda-7/IL-24 expression using stable transfection methods, compared with non-transfected cells and cells transfected with vector alone, this finding suggested that the growth suppression observed in these cells was not due to an increase in cell death. However, when Raji and Daudi cells were treated with CDDP, epirubicin and VCR, the percentage of Annexin V-FITC-positive cells was significantly higher in cells overexpressing Mda-7/IL-24 than in non-transfected and vector-transfected cells, and there was no significant difference between the latter two cultures. Those results indicate that apoptosis may not be involved in Mda-7/IL-24-induced growth inhibition of Raji and Daudi cells, but Mda-7/IL-24 promotes the apoptosis of Raji and Daudi cells treated with chemotherapeutic agents, so that Mda-7/IL-24 is a strong chemosensitizer for B cell lymphoma.

The multidrug-resistance genes MDR1, MRP1, BMII and Topo II may be involved in sensitivity of Mda-7/IL-24 overpressing cells to chemotherapy drugs. To address the underlying mechanism that may be responsible for Mda-7/IL-24-mediated chemosensitizing effect, we analyzed the changes of multidrug-resistance genes expression. Since MDR1, MRP1, BMI and Topo II are genes associated with the induction of multi-drug resistance, their expression was assessed in Raji and Daudi overexpressing Mda-7/IL-24. Our results showed that the levels of expression of MDR1, MRP1 and BMI1 mRNA and protein were reduced, whereas the levels of Topo II were increased, in lymphoma cells overexpressing Mda-7/IL-24 compared with both non-transfected and 

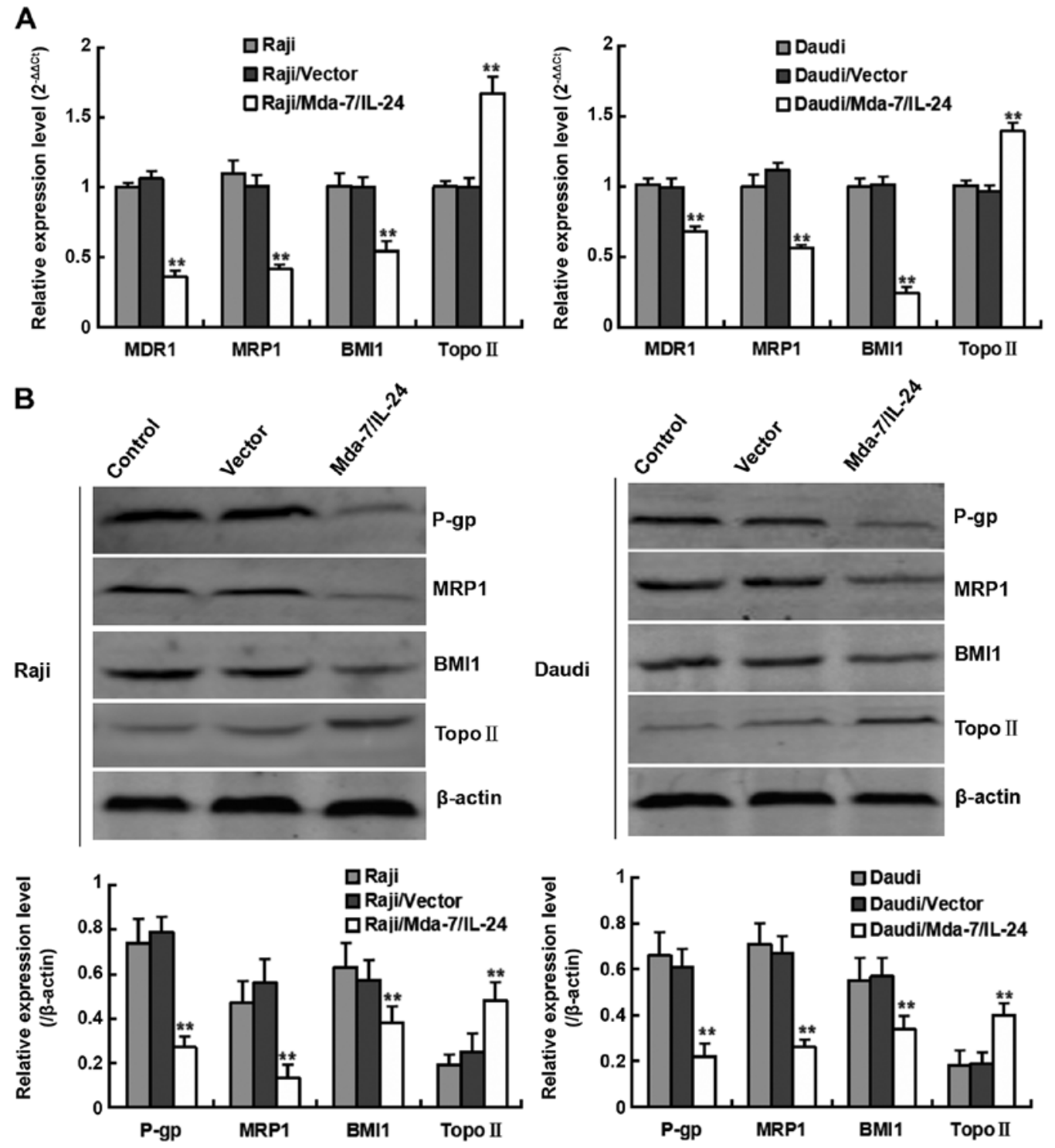

Figure 4. Effect of Mda-7/IL-24 on the expression of proteins related to chemotherapy sensitivity in Raji and Daudi cells. (A) RT-qPCR assays of the levels of expression of MDR1, MRP1, BMI1 and Topo II mRNAs in Raji and Daudi cells. (B) Western blot assays of the levels of P-glycoprotein (P-gp; encoded by MDR1), MRP1, BMI1 and Topo II protein expression in Raji and Daudi cells; $\beta$-actin was used as an internal loading control. The experiments shown are representative of three independent experiments. ${ }^{* *} \mathrm{P}<0.01$, compared with the control and vector groups.

vector-transfected cells (Fig. 4A and B). Those results indicate that the multidrug-resistance genes MDR1, MRP1, BMI1 and Topo II may be involved in sensitivity of overpressing cells to chemotherapy drugs.

To further determine the signaling pathway influenced by Mda-7/IL-24, the effects of Mda-7/IL-24 on the activity of ERK signal that is involved in regulating expression of MDR-related genes was evaluated. As shown in Fig. 5, the expression levels of total ERK1/2 and p-ERK1/2 were significantly decreased in Raji and Daudi overexpressing Mda-7/IL-24. It suggested that Mda-7/IL-24 can suppress the activity of ERK signaling pathway. Several reports have shown that MDR-related proteins including P-gp, MRP1 and BMI1 are positively regulated by the ERK pathway and blockade of the ERK pathway can suppress their expression (10-13). Thus, ERK signaling pathway may be a potential drug target for circumventing MDR. Furthermore, it is well known that GTP-RhoA signaling pathway is upstream of the ERK signaling pathway $(14,15)$.
Western blot assay showed that expression of GTP-RhoA was also influenced by Mda-7/IL-24 (Fig. 5).Taken together, the above data indicated that there is an axis of GTP-RhoA-ERK regulating expression of MDR-related genes MDR1, MRP1 and BMI1, but the mechanism that upregulates the expression of Topo II is still unknown.

Mda-7/IL-24 enhances the intracellular accumulation of Rhodamine-123 in lymphoma cells. The ability of Mda-7/IL-24 to inhibit the efflux of MDR-related proteins such as P-glycoprotein (P-gp) and MRP1 was assessed by Rhodamine-123 exclusion assays. The intracellular accumulation of Rhodamine-123 was significantly higher in Mda-7/IL-24 overexpressing cells than in non-transfected and vector-transfected cells (Fig. 6). These results suggested that Mda-7/IL-24 may reduce the pump effect of MDR-related proteins in Raji and Daudi cells, increasing the intracellular accumulation and reducing the efflux of chemotherapy drugs. 

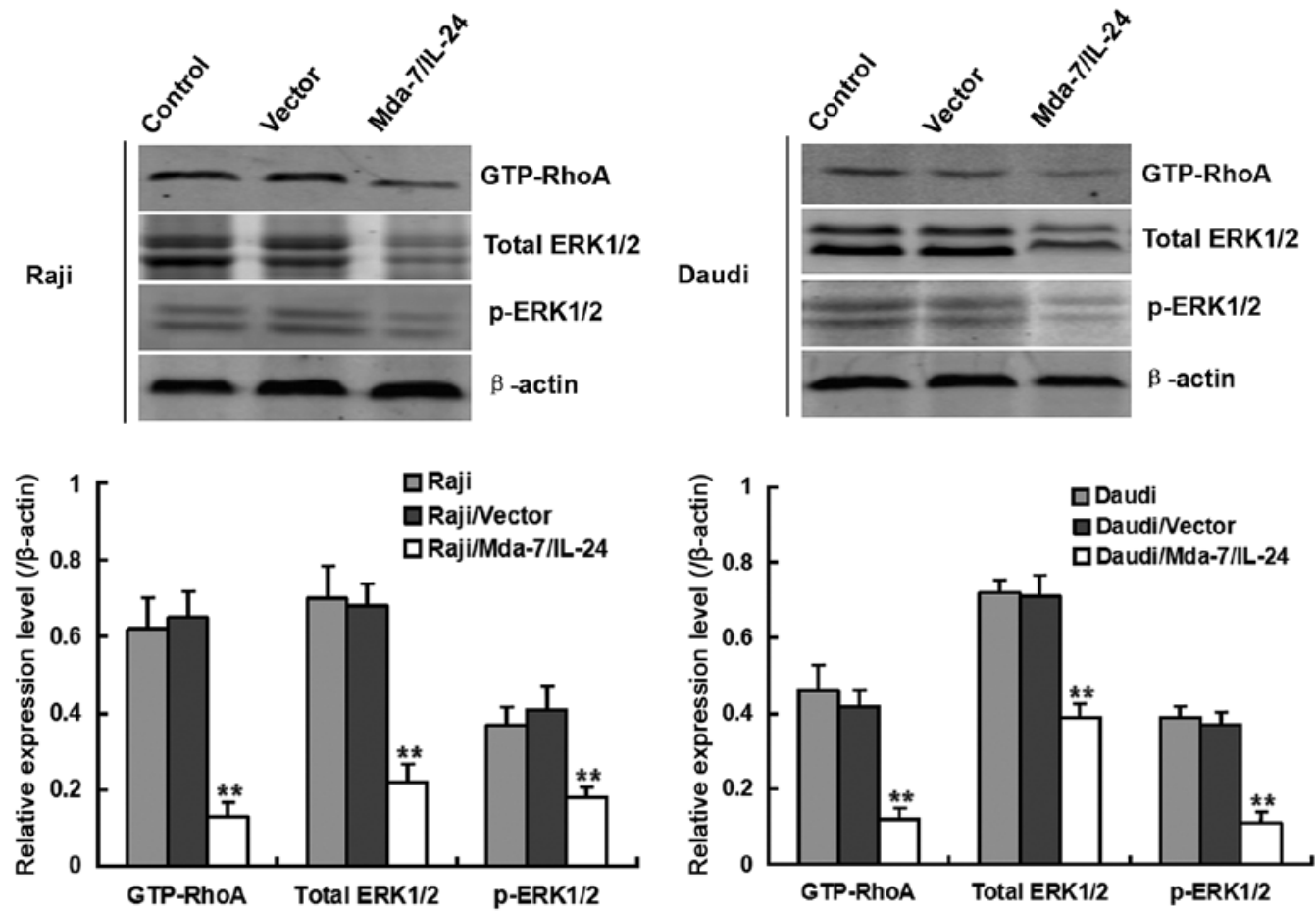

Figure 5. Effect of Mda-7/IL-24 on the expression of GTP-RhoA-ERK signaling pathway in Raji and Daudi cells. Western blot assays of the levels of GTP-RhoA, total ERK1/2 and p-ERK1/2 protein expression in Raji and Daudi cells; $\beta$-actin was used as an internal loading control. The experiments shown are representative of three independent experiments. ${ }^{* *} \mathrm{P}<0.01$, compared with the control and vector groups.
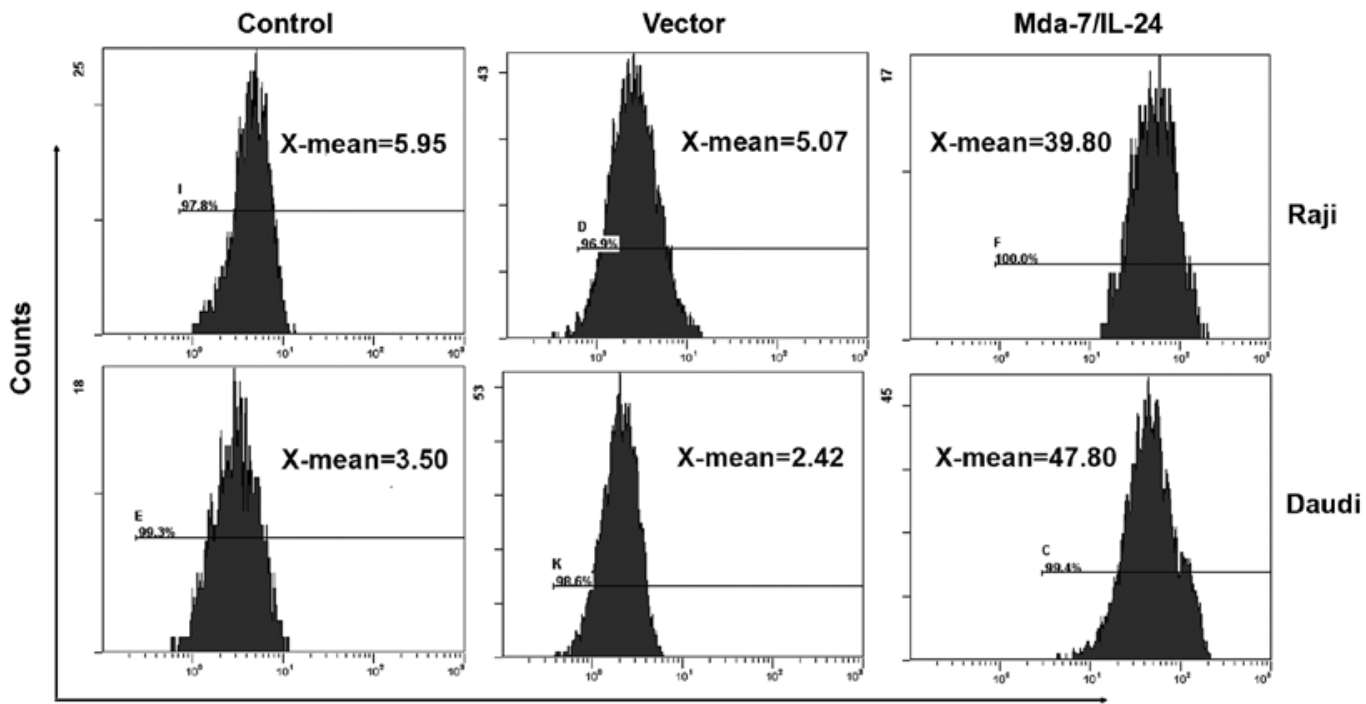

Rhodamine-123

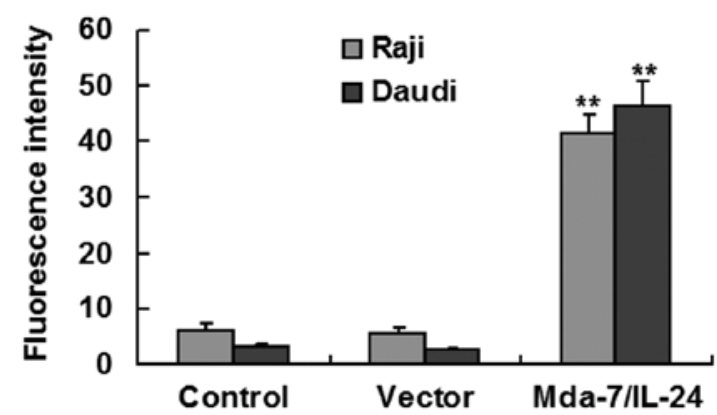

Figure 6. Effect of Mda-7/IL-24 on the intracellular accumulation of Rhodamine-123 in Raji and Daudi cells. Raji and Daudi cells were incubated with $5 \mathrm{mg} / \mathrm{ml}$ Rhodamine-123 for $45 \mathrm{~min}$, and intracellular fluorescence was measured to assess the pump function of MDR related protein. The fluorescence intensity indicated the intracellular concentration of Rhodamine-123, as shown on the histogram. Data presented are the mean \pm SD from three independent experiments. ${ }^{* *} \mathrm{P}<0.01$, compared with the control and vector groups. 


\section{Discussion}

B cell lymphoma is one of the most common types of hematopoietic tumors (16). Chemotherapy is the first-line choice for lymphoma treatment. However, tumors often become resistant to chemotherapy drugs during treatment. MDR can result in the failure of chemotherapy and the death of patients. Studies are required to elucidate the mechanisms of drug resistance, and new treatment strategies are needed to improve cure rates and survival in patients with lymphoma. Changes in molecular regulatory networks that increase anti-apoptotic activity and reduce the apoptosis of tumor cells are the primary mechanisms associated with resistance to chemotherapy drugs $(2,17)$. Methods of enhancing sensitivity to chemotherapy and avoiding MDR remain among the most difficult problems in tumor treatment.

Combinations of chemotherapy with gene therapy are promising in the treatment of B cell lymphoma (18). Although Mda-7/IL-24 has been widely regarded as an antitumor molecule and has been shown to inhibit hematopoietic tumor proliferation $(5,6)$, little is known about its effects on lymphoma cell sensitivity to chemotherapy. Recent studies have shown that Mda-7/IL-24 could reverse MDR in human colorectal and hepatocellular cancers $(2,19)$, but whether it plays a similar role in B cell lymphoma has not been determined. The present study is the first to show that transfection of endogenous Mda-7/IL-24 sensitized Raji and Daudi lymphoma cells to chemotherapy drugs by affecting factors related to chemotherapy drug resistance.

In the present study, we first assessed the chemosensitizing effects of Mda-7/IL-24 gene in B lymphoma cells in vitro. MTS assay results showed that Mda-7/IL-24 significantly retarded proliferation of Raji and Daudi cells, and enhanced the sensitivity of these lymphoma cells to various chemotherapy drugs, including CDDP, epirubicin and VCR, and that $\mathrm{IC}_{50}$ was lower in cells transfected with Mda-7/IL-24 than in non-transfected and vector-transfected cells. These results were in good agreement with the effects of Mda-7/IL-24 on these types of tumor cells $(2,5,6)$. In addition, although the apoptosis-inducing capability of Mda-7/IL-24 has been widely documented in various solid tumors, we did not observe significant induction of apoptosis in the two lymphoma cell lines. However, flow cytometry results showed that transfecting Mda-7/IL-24 enhanced the apoptotic rates in cells treated with these chemotherapeutic agents. The effect that Mda-7/IL-24 plus chemotherapy drugs enhanced the induction of apoptosis in Raji and Daudi cells indicating that Mda-7/IL-24 could be a potent model of adjuvant chemotherapy for B cell lymphoma.

To study the mechanisms that may be responsible for Mda-7/IL-24-mediated chemosensitizing effect, we analyzed the changes of expression in various multidrug-resistance genes. Abnormal levels of expression of MDR1, MRP1, BMI1 and Topo II were found to be important in impairing tumor chemosensitivity, resulting in MDR (20-23). P-gp is the best-known and principal mediator of MDR. P-gp, which is encoded by MDR1, is involved in pumping chemotherapy drugs from the inside to the outside of tumor cells, and in preventing intracellular accumulation of these drugs $(20,24)$. MRP1 functions similarly to MDR1 in reducing tumor sensitivity to chemotherapy, and it can recognize and pump out chemotherapy drugs bound to glutathione in tumor cells. High expression of MDR1 and MRP1 in different types of B cell lymphoma tissues has been associated with poor response to chemotherapy (25). Thus, our finding, that Mda-7/IL-24 overexpression downregulated MDR1 and MRP1 expression in Raji and Daudi cells, suggests that Mda-7/IL-24 may enhance the intracellular accumulation of anticancer drugs.

BMI1, which is essential for the self-renewal of normal and malignant stem cells, is another important gene that induces MDR. The mechanism of action of BMI1 may be related to antioxidation and the upregulation of bcl-2, resulting in resistance to chemotherapy-induced apoptosis $(26,27)$. Downregulation of BMI1 may increase drug-induced tumor cell apoptosis, thus enhancing sensitivity to chemotherapy (28). Topo II, which can bind to chemotherapeutic agents, with binding of these drugs to the $\alpha$ chain of Topo II inhibiting the proliferation and duplication of DNA in tumor cells (17). Upregulating the expression of Topo II by transfection has been reported to enhance the cytotoxicity of chemotherapy drugs $(23,29)$. Thus, the ability of Mda-7/IL-24 to enhance Topo II expression in Raji and Daudi suggests that it may enhance sensitivity to chemotherapy in vivo. Collectively, all of these findings suggest that Mda-7/IL-24 enhances sensitivity to chemotherapy via various targets, with the changes in MDR related protein expression, enhancing the cytotoxic effects of chemotherapy agents on lymphoma cells.

To further investigate the molecular mechanism of Mda-7/ IL-24-induced chemosensitizing effect, we analyzed the activities of ERK and GTP-RhoA signaling pathways. It is known that ERK plays an important role in positively regulating expression of various MDR-related genes including MDR1, MRP1 and BMI1 (10-13). A previous report showed that decreased activities of ERK1/2 was detected in Human leukemia U937 and HL60 cells overpressing Mda-7/IL-24 (4). In the present study, overpressing Mda-7/IL-24 also resulted in a similar result in Raji and Daudi cells. Moreover, GTP-RhoA is known as upstream activator of cell ERK pathway, and it was also reported to be related to induction of MDR (30). In the present study, we found that decreased GTP-RhoA in Mda-7/IL-24 overpressing lymphoma cells indicated that GTP-RhoA may be a key factor affected by Mda-7/IL-24 regulating the expression of MDR-related genes.

In addition, Mda-7/IL-24 overexpression also significantly increased the intracellular accumulation of Rhodamine-123 in Raji and Daudi cells, suggesting that Mda-7/IL-24 impaired the pump function of MDR-related proteins.

In summary, we found that Mda-7/IL-24 can enhance the sensitivity of B lymphoma cells to chemotherapy by decreasing the expression of MDR1, MRP1 and BMI1 via GTP-RhoA-ERK signaling pathway, and by increasing the expression of Topo II and the intracellular accumulation of anticancer drugs. In addition, Mda-7/IL-24 could increase the apoptosis rate of $\mathrm{B}$ lymphoma cells treated with chemotherapy drugs. Mda-7/IL-24 may therefore be a potential candidate for treatment of MDR in B cell lymphomas.

\section{Acknowledgements}

The present study was supported by a grant from the National Natural Science Foundation of China (no. 81173611). 


\section{References}

1. Morin RD, Mendez-Lago M, Mungall AJ, Goya R, Mungall KL, Corbett RD, Johnson NA, Severson TM, Chiu R, Field M, et al: Frequent mutation of histone-modifying genes in non-Hodgkin lymphoma. Nature 476: 298-303, 2011.

2. Fang P, Zhang X, Gao Y, Ding CR, Cui F and Jiao SC: Reversal effect of melanoma differentiation associated gene-7/interleukin-24 on multidrug resistance in human hepatocellular carcinoma cells. Anat Rec 295: 1639-1646, 2012.

3. Huo W, Li ZM, Zhu XM, Bao YM and An LJ: MDA-7/IL-24 suppresses tumor adhesion and invasive potential in hepatocellular carcinoma cell lines. Oncol Rep 30: 986-992, 2013.

4. Rahmani M, Mayo M, Dash R, Sokhi UK, Dmitriev IP, Sarkar D, Dent P, Curiel DT, Fisher PB and Grant S: Melanoma differentiation associated gene-7/interleukin-24 potently induces apoptosis in human myeloid leukemia cells through a process regulated by endoplasmic reticulum stress. Mol Pharmacol 78: 1096-1104, 2010.

5. Dong CY, Zhang F, Duan YJ, Yang BX, Lin YM and Ma XT: mda-7/IL-24 inhibits the proliferation of hematopoietic malignancies in vitro and in vivo. Exp Hematol 36: 938-946, 2008

6. Qian W, Liu J, Tong Y, Yan S, Yang C, Yang M and Liu X: Enhanced antitumor activity by a selective conditionally replicating adenovirus combining with MDA-7/interleukin-24 for B-lymphoblastic leukemia via induction of apoptosis. Leukemia 22: 361-369, 2008.

7. Sahoo A and Im SH: Molecular mechanisms governing IL-24 gene expression. Immune Netw 12: 1-7, 2012.

8. Gopalan B, Shanker M, Chada S and Ramesh R: MDA-7/IL-24 suppresses human ovarian carcinoma grow th in vitro and in vivo. Mol Cancer 6: 11, 2007.

9. Pataer A, Vorburger SA, Barber GN, Chada S, Mhashilkar AM, Zou-Yang H, Stewart AL, Balachandran S, Roth JA, Hunt KK, et al: Adenoviral transfer of the melanoma differentiationassociated gene 7 (mda7) induces apoptosis of lung cancer cells via up-regulation of the double-stranded RNA-dependent protein kinase (PKR). Cancer Res 62: 2239-2243, 2002.

10. Yan F, Bai LP, Gao H, Zhu CM, Lin L and Kang XP: EGF reverses multi-drug resistance via the p-ERK pathway in HepG2/ADM and SMMC7721/ADM hepatocellular carcinoma models. Asian Pac J Cancer Prev 15: 2619-2623, 2014.

11. Wang NN, Zhao LJ, Wu LN, He MF, Qu JW, Zhao YB, Zhao WZ, Li JS and Wang JH: Mechanistic analysis of taxol-induced multidrug resistance in an ovarian cancer cell line. Asian Pac J Cancer Prev 14: 4983-4988, 2013.

12. Yan F, Wang XM, Pan C and Ma QM: Down-regulation of extracellular signal-regulated kinase $1 / 2$ activity in P-glycoprotein-mediated multidrug resistant hepatocellular carcinoma cells. World J Gastroenterol 15: 1443-1451, 2009.

13. Suh HN and Han HJ: Collagen I regulates the self-renewal of mouse embryonic stem cells through $\alpha 2 \beta 1$ integrin- and DDR1dependent Bmi-1. J Cell Physiol 226: 3422-3432, 2011.

14. Jayakumar T, Chiu CC, Wang SH, Chou DS, Huang YK and Sheu JR: Anti-cancer effects of CME-1, a novel polysaccharide, purified from the mycelia of Cordyceps sinensis against B16-F10 melanoma cells. J Cancer Res Ther 10: 43-49, 2014.

15. Sánchez-Mir L, Soto T, Franco A, Madrid M, Viana RA, Vicente J, Gacto M, Pérez P and Cansado J: Rhol GTPase and PKC ortholog Pck1 are upstream activators of the cell integrity MAPK pathway in fission yeast. PLoS One 9: e88020, 2014.

16. Chen S, Wang Z, Dai X, Pan J, Ge J, Han X, Wu Z, Zhou X and Zhao T: Re-expression of microRNA-150 induces EBV-positive Burkitt lymphoma differentiation by modulating c-Myb in vitro. Cancer Sci 104: 826-834, 2013.
17. Xu Y, Zheng H, Kang JS, Zhang L, Su J, Li HY and Sun LK: 5-Nitro-2-(3-phenylpropylamino) benzoic acid induced drug resistance to cisplatin in human erythroleukemia cell lines. Anat Rec 294: 945-952, 2011.

18. Zhu X, Ma Y and Liu D: Novel agents and regimens for acute myeloid leukemia: 2009 ASH annual meeting highlights. J Hematol Oncol 3: 17, 2010.

19. Emdad L, Lebedeva IV, Su ZZ, Sarkar D, Dent P, Curiel DT and Fisher PB: Melanoma differentiation associated gene-7/ interleukin-24 reverses multidrug resistance in human colorectal cancer cells. Mol Cancer Ther 6: 2985-2994, 2007.

20. Loo TW, Bartlett MC, Shi L and Clarke DM: Corrector-mediated rescue of misprocessed CFTR mutants can be reduced by the P-glycoprotein drug pump. Biochem Pharmacol 83: 345-354, 2012.

21. Wang E, Bhattacharyya S, Szabolcs A, Rodriguez-Aguayo C, Jennings NB, Lopez-Berestein G, Mukherjee P, Sood AK and Bhattacharya R: Enhancing chemotherapy response with Bmi-1 silencing in ovarian cancer. PLoS One 6: e17918, 2011.

22. Baird NJ, Fang XW, Srividya N, Pan T and Sosnick TR: Folding of a universal ribozyme: The ribonuclease P RNA. Q Rev Biophys 40: 113-161, 2007.

23. Tokiniwa H, Horiguchi J, Takata D, Kikuchi M, Rokutanda N, Nagaoka R, Sato A, Odawara H, Tozuka K, Oyama T, et al: Topoisomerase II alpha expression and the Ki-67 labeling index correlate with prognostic factors in estrogen receptor-positive and human epidermal growth factor type-2-negative breast cancer. Breast Cancer 19: 309-314, 2012.

24. Lu D, Shi HC, Wang ZX, Gu XW and Zeng YJ: Multidrug resistance-associated biomarkers PGP, GST-pi, Topo-II and LRP as prognostic factors in primary ovarian carcinoma. Br J Biomed Sci 68: 69-74, 2011.

25. Ohsawa M, Ikura Y, Fukushima H, Shirai N, Sugama Y, Suekane T, Hirayama M, Hino M and Ueda M: Immunohistochemical expression of multidrug resistance proteins as a predictor of poor response to chemotherapy and prognosis in patients with nodal diffuse large B-cell lymphoma. Oncology 68: 422-431, 2005.

26. Crea F, Duhagon Serrat MA, Hurt EM, Thomas SB, Danesi R and Farrar WL: BMI1 silencing enhances docetaxel activity and impairs antioxidant response in prostate cancer. Int J Cancer 128: 1946-1954, 2011.

27. Siddique HR, Parray A, Tarapore RS, Wang L, Mukhtar H, Karnes RJ, Deng Y, Konety BR and Saleem M: BMI1 polycomb group protein acts as a master switch for growth and death of tumor cells: Regulates TCF4-transcriptional factor-induced BCL2 signaling. PLoS One 8: e60664, 2013.

28. Zhu D, Wan X, Huang H, Chen X, Liang W, Zhao F, Lin T, Han J and Xie W: Knockdown of Bmil inhibits the stemness properties and tumorigenicity of human bladder cancer stem cell-like side population cells. Oncol Rep 31: 727-736, 2014.

29. Zhu WY, Hunag YY, Liu XG, He JY, Chen DD, Zeng F, Zhou JH and Zhang YK: Prognostic evaluation of CapG, gelsolin, P-gp, GSTP1, and Topo-II proteins in non-small cell lung cancer. Anat Rec 295: 208-214, 2012

30. Rigoni M, Riganti C, Vitale C, Griggio V, Campia I, Robino M, Foglietta M, Castella B, Sciancalepore P, Buondonno I, et al: Simvastatin and downstream inhibitors circumvent constitutive and stromal cell-induced resistance to doxorubicin in IGHV unmutated CLL cells. Oncotarget 6: 29833-29846, 2015. 